\title{
The Impact of Special Access Programs (SAP) on Prescribing Patterns in Metastatic Castration Resistant Prostate Cancer (mCRPC)
}

\author{
Nimira Alimohamed ${ }^{1}$, Raya Leibowitz-Amit ${ }^{2}$, Arnoud Templeton ${ }^{3}$, Jo-An Seah ${ }^{4}$, \\ Francisco Emilio Vera-Badillo ${ }^{5}$, Anthony Michael Joshua ${ }^{6}$, Sarah Elizabeth Wong ${ }^{7}$, \\ Jennifer Jane Knox ${ }^{7}$, Ian Frederick Tannock ${ }^{7}$, Srikala Sujata Sridhar, * \\ ${ }^{1}$ Department of Medicine, Tom Baker Cancer Centre, Calgary, Canada \\ ${ }^{2}$ Department of Medicine, Sheba Medical Center, Tel-Hashomer, Israel \\ ${ }^{3}$ Department of Oncology, Claraspital, Basel, Switzerland \\ ${ }^{4}$ St Vincent's Hospital, Melbourne, Australia \\ ${ }^{5}$ Department of Oncology, Queen's University, Kingston, Canada \\ ${ }^{6}$ Department of Medical Oncology, St Vincent's Private Hospital, Sydney, Australia \\ ${ }^{7}$ Department of Medicine, Princess Margaret Cancer Center, Toronto, Canada
}

Email address:

srikala.sridhar@uhn.ca (S. S. Sridhar)

${ }^{*}$ Corresponding author

To cite this article:

Nimira Alimohamed, Raya Leibowitz-Amit, Arnoud Templeton, Jo-An Seah, Francisco Emilio Vera-Badillo, Anthony Michael Joshua, Sarah Elizabeth Wong, Jennifer Jane Knox, Ian Frederick Tannock, Srikala Sujata Sridhar. The Impact of Special Access Programs (SAP) on Prescribing Patterns in Metastatic Castration Resistant Prostate Cancer (mCRPC). International Journal of Clinical Oncology and Cancer Research. Vol. 6, No. 1, 2021, pp. 33-37. doi: 10.11648/j.ijcocr.20210601.15

Received: January 26, 2021; Accepted: February 13, 2021; Published: February 27, 2021

\begin{abstract}
Background: Special access programs provide physicians in Canada access to new drugs before they are publicly funded, but little is known about how they impact prescribing practices. For men with metastatic castration-resistant prostate cancer post-docetaxel chemotherapy, a special access program for enzalutamide was open from June-December 2013. To better understand how this impacted prescribing practices, we surveyed medical oncologists at our institution. Methods: All four genitourinary medical oncologists at the Princess Margaret Cancer Centre completed an anonymous paper or electronic survey for each patient enrolled. The survey consisted of seven multiple-choice questions (with free text option) asking about disease characteristics, prior treatments, and reasons for prescribing enzalutamide. Results: Surveys were completed on all 155 patients; $92 \%(142 / 155)$ had metastatic disease, with 20\% (28/142) having visceral disease; 8\% (13/155) had non-metastatic disease; and the majority (92\%) had progressive disease. All patients were on androgen deprivation therapy, $60 \%$ had prior prednisone, $30 \%$ had prior abiraterone, and 34\% had prior docetaxel. Most (50\%) used enzalutamide because it was supported by available data; $35 \%$ reported free drug was the motivating factor; $10 \%$ indicated their patients were unfit for chemotherapy; and in 5\%, all other options were exhausted. Over half reported feeling enzalutamide was the appropriate treatment option for their patient at that time. Conclusions: During the special access program, most patients received enzalutamide in settings supported by available evidence. A minority did, however, received enzalutamide outside of the formally studied setting, suggesting that funding and accessibility can impact prescribing practices.
\end{abstract}

Keywords: Prostatic Neoplasms, Medical Oncology, Special Access Programs, Enzalutamide 


\section{Introduction}

In Canada, novel therapeutics are regulated by Health Canada (HC) through the Food and Drug Act $[1,2]$. Once a new drug is approved, a notice of compliance and a drug identification number are assigned. The Pan-Canadian Oncology Drug Review then evaluates for clinical benefit, pricing is negotiated by the pan-Canadian Pharmaceutical Alliance, and finally, each province makes its own funding decision [3]. From initial submission to HC, approval takes approximately 12 months, with an additional 17 months on average for a drug to appear on the provincial formulary. This can be contrasted with the US where time from initial submission to FDA approval and drug availability is only about 9 months [4].

Delays in access to new drugs can negatively impact patient outcomes. During the long interval from HC approval to drug access, physicians may be able to access new drugs through clinical trials, private insurance plans, or special access programs (SAP). While practical, legal, and ethical issues have arisen from these SAPs [5], they have been used extensively to facilitate access to new oncology drugs. One study of Canadian oncologists evaluating methods to overcome barriers to accessing unfunded anticancer drugs showed that $96 \%$ of respondents use SAPs [6].

From June-December 2013, a SAP was established for the drug enzalutamide, a well-tolerated androgen-receptor blocker, for men with metastatic castration-resistant prostate cancer after failure of docetaxel chemotherapy. This was based on the interim analysis of the AFFIRM randomized phase 3 trial which showed enzalutamide had a 5-month survival benefit over placebo, ultimately leading to its $\mathrm{HC}$ approval in May 2013 [7]. During the SAP, results of another randomized phase 3 trial, PREVAIL, were announced [8]. In PREVAIL, enzalutamide also showed a survival benefit in the pre-docetaxel setting but was not $\mathrm{HC}$ approved for this indication until April 2015 [9]. More recently, the ENZAMET and ARCHES studies also reported a survival benefit for enzalutamide when added to ADT in the metastatic hormone sensitive setting $[10,11]$; this indication has received HC approval but is not yet funded in Canada.

Although SAPs are used quite commonly to access new drugs in oncology, the impact of SAPs on prescribing practices is not well understood. We hypothesized that prescribing patterns may be influenced by drug availability and perceived benefit for additional indications, beyond the setting where there was HC approval. This hypothesis was tested by surveying medical oncologists offering patients enzalutamide via the SAP.

\section{Methods}

The enzalutamide SAP was available for men with prostate cancer treated at the Princess Margaret Cancer Centre (Toronto, Canada) from June to December 2013. During this time, medical oncologists were approached to complete an anonymous paper or electronic survey for each patient they enrolled in the program. Patient identifying information was not collected for the survey, and physicians completed these surveys anonymously.

Respondents were asked to comment on whether the patient had metastatic or non-metastatic prostate cancer, the main sites of metastatic disease, and if there was disease progression at the time of starting enzalutamide. Information on prior treatment was also obtained. The reasons for prescribing enzalutamide at that time were also explored. Possible reasons included:

1) enzalutamide is only available to patients at no cost for the next few weeks/months,

2) the use of enzalutamide is supported by the data,

3) the patient is not fit for chemotherapy,

4) the patient has exhausted all other treatment options.

Finally, physicians were asked to indicate their primary motivation for prescribing enzalutamide, funding and accessibility issues aside:

1) enzalutamide is the most appropriate treatment at this point in time,

2) enzalutamide is as good an option as other approved lines of treatment at this point in time,

3) enzalutamide would be prescribed as the next line of treatment but at further progression

4) enzalutamide would not be the next line of treatment.

Surveys were completed shortly after the patients were enrolled on the program.

\section{Results}

From June to December 2013, 155 patients were prescribed enzalutamide through the SAP by genitourinary medical oncologists at our institution. Surveys were completed for all 155 patients.

\subsection{Disease Characteristics}

Characteristics of the patients are shown in Table 1. All patients had received a prior LHRH agonist (100\%) and were on continuous anti-androgen therapy (100\%). Patients had received multiple prior treatments including: 93/155 (60\%) prior prednisone, 56/155 (36\%) prior abiraterone acetate (28 pre-docetaxel chemotherapy, 28 post- docetaxel chemotherapy), and 53/155 (34\%) prior docetaxel. 102/155 (66\%) had not received prior docetaxel. Of the $66 \%$ who did not receive prior docetaxel, approximately $50 \%$ received enzalutamide before the data showing a benefit from enzalutamide in the pre-docetaxel setting were released in October 2013.

\subsection{Reasons for Enzalutamide Use}

Half of the surveys indicated that use of enzalutamide was supported by the AFFIRM data (in the post-docetaxel setting) (Figure 1). The remainder of responses indicated that enzalutamide was prescribed because it was available to patients at no cost $(35 \%)$; that the patient was not fit for chemotherapy $(10 \%)$; or that the patient had exhausted all other treatment options $(5 \%)$. 


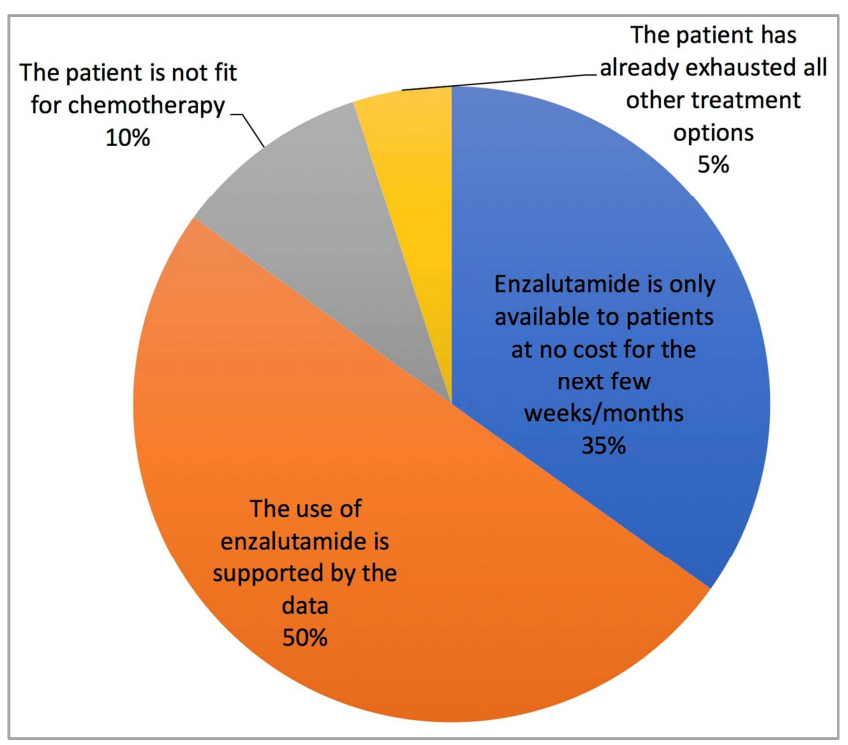

Figure 1. Responses to question; "What is your primary reason for prescribing enzalutamide?"

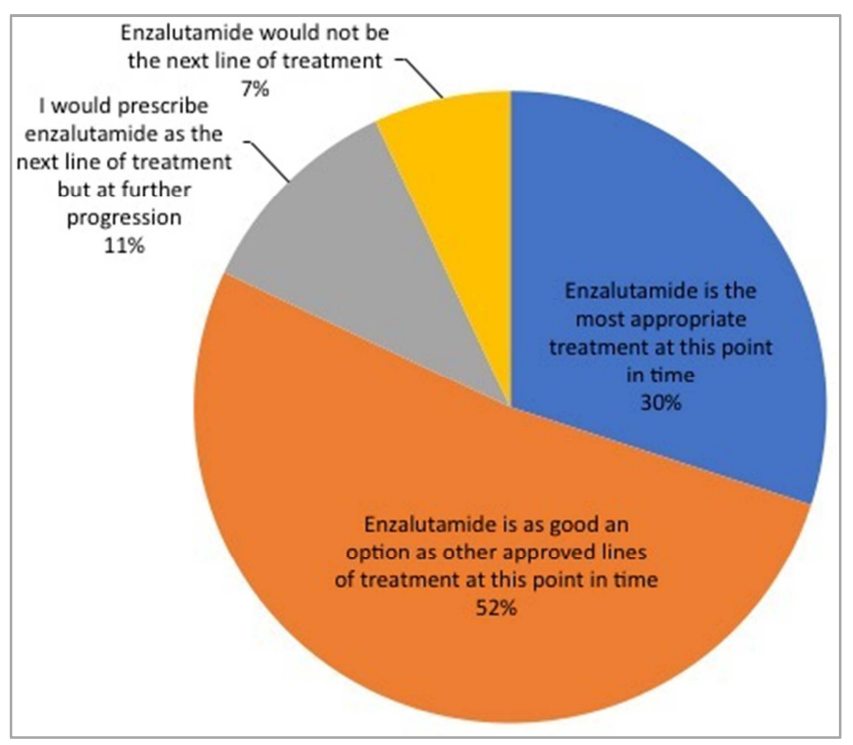

Figure 2. Responses to the question; "Funding and accessibility issues aside, why are you prescribing enzalutamide at this time?"

Table 1. Patient and Disease Characteristics.

\begin{tabular}{lll}
\hline Survey Question & Patient and Disease Characteristics & \% of patients $(\mathbf{N} / \mathbf{1 5 5})$ \\
\hline Does this patient have metastatic castration resistant prostate & Non-metastatic Disease & $8 \%(13 / 155)$ \\
cancer (mCRPC) now? & Metastatic Disease & $92 \%(142 / 155)$ \\
Does this patient have progressive disease now, at the time of & Progressive Disease & $92 \%(142 / 155)$ \\
starting enzalutamide? & No Progression & $8 \%(13 / 155)$ \\
& Bone Only & $46 \%(65 / 142)$ \\
& Bone + Lymph Node & $21 \%(28 / 142)$ \\
What are the sites of metastatic disease in this patient? & Visceral Disease & $20 \%(28 / 142)$ \\
& Lymph Node Only & $8 \%(13 / 42)$ \\
& Other & $4 \%(6 / 142)$ \\
& LHRH ongoing & $100 \%(155 / 155)$ \\
What previous treatments has this patient had? & Prior prednisone & $60 \%(93 / 155)$ \\
& Prior abiraterone & $36 \%(56 / 155)$ \\
& Pre-chemotherapy & $50 \%(28 / 56)$ \\
& Post-chemotherapy & $50 \%(28 / 56)$ \\
& Prior docetaxel & $34 \%(53 / 155)$ \\
\hline
\end{tabular}

Physicians were asked to select the most appropriate statement regarding the choice to use enzalutamide if funding and accessibility were not concerns. The majority stated that enzalutamide is as good an option as other approved lines of treatment (Figure 2). However, 7\% of responses indicated that had it not been for the SAP, enzalutamide would not have been the preferred next line of therapy, and $11 \%$ of responses indicated that enzalutamide would have been used but at the time of further disease progression.

\section{Discussion}

SAPs play a critical role in ensuring that patients have timely access to novel therapeutics. The time from initial release of positive trial results to the time a drug is available can approach 2 years or more and varies across the country [4]. During this time, physicians often look for SAPs or clinical trials, or sometimes, patients will pay privately or attempt to access drugs out of the province or even country. SAPs are designed to be less stringent than clinical trials and may offer important insights and safety information about how a drug performs in the general population $[12,13]$. With the rising costs of both oncology drugs and clinical trials, SAPs may play a bigger role in the field of oncology. However, very little is known about how SAPs impact prescribing practices.

In this study, we found, as expected, that the majority of patients had mCRPC. However, only $34 \%$ had prior docetaxel and fit the eligibility criteria for the AFFIRM Phase 3 trial. Interestingly, 66\% had mCRPC but had not received prior docetaxel, and half of these patients were treated with enzalutamide prior to the release of PREVAIL trial results. Likewise, a recent Canadian study of the CheckMate 128 SAP for nivolumab plus ipilimumab in advanced melanoma revealed differences between the SAP population and the patient population of the original randomized controlled trials, CheckMate 069 and CheckMate 067. Specifically, the SAP included patients that had received other prior systemic treatment, whereas CheckMate 069 and Checkmate 067 did not [13]. Another SAP, the KEYNOTE- 
030, which provided access to pembrolizumab in advanced melanoma, also expanded eligibility criteria compared to its preceding clinical trials, as it aimed to provide treatment access prior to commercial availability rather than contribute as another clinical trial [14].

There were likely several reasons to explain our study's results. First, the SAP did not mandate that patients had to have metastatic disease and had to have prior docetaxel. Second, the majority of patients did not have visceral disease, and so there may have been less incentive to offer docetaxel chemotherapy if enzalutamide was available. Third, enzalutamide is a well-tolerated oral drug with few side effects, and in this elderly patient population, it may have been the preferred option over docetaxel chemotherapy. Fourth, all the medical oncologists surveyed participated in the PREVAIL or AFFIRM trials and had prior experience with enzalutamide. They were therefore familiar with its efficacy and any potential safety and monitoring issues, and they felt comfortable prescribing it in the pre-docetaxel setting, even prior to the release of the PREVAIL results. It is unclear if the same trend would have been seen for a chemotherapy drug, or one where there was less familiarity a priori.

Interestingly, most physicians indicated that enzalutamide was the best or as good as other available options at the time, but $7 \%$ indicated that enzalutamide would not have been their next line of treatment had it not been for the SAP. When asked for reasons for prescribing enzalutamide, $35 \%$ indicated that a reason was that enzalutamide was available at no cost through the SAP. This result is similar to that of a previous Australian study of SAPs in colorectal cancer, which also found that economic reasons influenced SAP participation [15]. This serves to highlight that SAPs have the potential to change a treatment decision, especially if there is concern that access to a drug will subsequently become limited. Many SAPs are open for a finite period of time, pending a funding decision, thus leading to potential lapses in drug availability between the end of a SAP and drug availability. In this setting, clinical trials may be able to address access issues and at the same time collect additional safety information or information on patients that did not strictly fit the trial criteria.

This study provides important insights into prescribing practices on an SAP but has some key limitations. This study was conducted at a single, large, academic institution with dedicated genitourinary medical oncologists who already had some experience with enzalutamide. The surveys were done after patients were enrolled on the SAP and not in real time, so there may have been potential for recall bias. Finally, enzalutamide is an oral, well-tolerated drug, and findings may not be generalizable to other drugs with higher potential for toxicities.

\section{Conclusion}

During the SAP, most patients received enzalutamide in settings supported by available evidence. However, a minority received enzalutamide outside of the formally studied setting, and a minority of physicians indicated that enzalutamide would not have been their next line of treatment had it not been for the SAP, suggesting that funding and accessibility can impact prescribing practices. When it comes to new drugs in oncology, strong, collaborative relationships are required between regulatory bodies, industry partners, physicians, and patient advocates to ensure that drug access occurs in a timely manner. Although SAPs aim to fill the gap between HC approval and drug access, these stakeholder groups must also be aware of and mitigate any potential biases which may arise during these programs. Ultimately, these combined efforts should optimize patient outcomes with earlier access to novel effective therapeutics.

\section{Conflict of Interest}

N. Alimohamed has served as an advisory board member of Merck, Astellas, Pfizer, AstraZeneca, and Janssen. R. Leibowitz-Amit has served as an advisory board member of Pfizer, Bristol Meyers Squibb, MSD, Roche, Isotopia, AstraZeneca, Bayer, Astellas, and Janssen. A. Templeton has served as an advisory board member of Roche, Janssen, Astellas, MSD, Bristol Meyers Squibb, and Sanofi and has received honorary payment for lectures from Astellas, Janssen, and MSD. A. M. Joshua has contributed financially to Astellas and has participated in the ENZAMET trial with Astellas. J. J. Knox has received grants from Ibsen, Merck, and Roche for investigator-initiated trials and has participated in multiple trials for Bristol Myers Squibb, NuCana, AstraZeneca, Tahai, ArQule, and Merck. I. F. Tannock has received payment from Johnson and Johnson and Roche outside of submitted work. S. S. Sridhar has served as an advisory board member of Pfizer, Merck, Roche, Bristol Myers Squibb, Bayer, AstraZeneca, Astellas, Janssen, and Immunomedex and has participated in clinical trials with Pfizer, Merck, AstraZeneca, and Bayer. J. A. Seah, F. E. Vera-Badillo, and S. E. Wong declare that they have no competing interests.

\section{References}

[1] Government of Canada. Canada Health Act (R. S. C., 1985, c. C-6). http://laws-lois.justice.gc.ca/eng/acts/C-6/FullText.html. Accessed June 15, 2017.

[2] Government of Canada. Food and Drug Act (R. S. C., 1985, c. F-27). http://laws-lois.justice.gc.ca/eng/acts/F-27/. Accessed June 15, 2017.

[3] Pan-Canadian Oncology Drug Review. https://www.cadth.ca/pcodr. Accessed June 15, 2017.

[4] D. A. Ezeife, T. H. Truong, D. Y. C. Heng, et al, "Comparison of oncology drug approval between Health Canada and the US Food and Drug Administration," Cancer, vol. 121, pp. 168893, 2015.

[5] J. J. Darrow, A. Sarpatwari, J. Avorn, et al, "Practical, legal, and ethical issues in expanded access to investigational drugs," N Engl J Med, vol. 372, pp. 279-86, 2015. 
[6] D. Han, M. Trinkaus, S. Hogeveen, et al, "Overcoming obstacles in accessing unfunded oral chemotherapy; physician experience and challenges," J Oncol Pract, vol. 9, pp. 188-93, 2013.

[7] H. I. Scher, K. Fizazi, F. Saad, et al, "Increased survival with enzalutamide in prostate cancer after chemotherapy," N Engl J Med, vol. 367, pp. 1187-97, 2012.

[8] Astellas News Release October 22, 2013. https://www.astellas.com/en/corporate/news/detail/post.html. Accessed June 16, 2017.

[9] T. M. Beer, A. J. Armstrong, D. E. Rathkopf, et al, "Enzalutamide in metastatic prostate cancer before chemotherapy,” N Engl J Med, vol. 371, pp. 424-33, 2014.

[10] I. D. Davis, A. J. Martin, M. R. Stockler, et al, "Enzalutamide with standard first-line therapy in metastatic prostate cancer," N Engl J Med, vol. 381, pp. 121-31, 2019.

[11] A. J. Armstrong, R. Z. Szmulewitz, D. P. Petrylak, et al,
"ARCHES: a randomized, phase III study of androgen deprivation therapy with enzalutamide or placebo in men with metastatic hormone-sensitive prostate cancer," J Clin Oncol, vol. 37, pp. 2974-86, 2019.

[12] J. Martinalbo, D. Bowen, J. Camarero, et al, "Early market access of cancer drugs in the EU," Ann Oncol, vol. 27, pp. 96$105,2015$.

[13] D. Hogg, J. G. Monzon, S. Ernst, et al, "Canadian cohort expanded-access program of nivolumab plus ipilimumab in advanced melanoma," Curr Oncol, vol. 27, pp. 204-14, 2020.

[14] T. C. Gangadhar, W. J. Hwu, M. A. Postow, et al, "Efficacy and safety of pembrolizumab in patients enrolled in KEYNOTE-030 in the United States: an expanded access program," J Immunother, vol. 40, pp. 334-40, 2017.

[15] J. Tie, P. Gibbs, "Treatment with unfunded drugs in oncology: the impact of access programmes and clinical trials," Intern Med J, vol. 43, pp. 23-31, 2013. 